\title{
Evaluation of Hybrid Topologies under Mobility of ZigBee Devices using Different Trajectories
}

\author{
Ranjeet Singh \\ M.Tech Scholar, ECE \\ SBSSTC, Moga Road \\ Ferozepur - 152004
}

\author{
Jaswinder Kaur \\ Assistant Professor, ECE \\ SBSSTC, Moga Road \\ Ferozepur - 152004
}

\author{
Inderjeet Singh Gill \\ Assistant Professor, ECE \\ SBSSTC, Moga Road \\ Ferozepur - 152004
}

\begin{abstract}
Wireless Sensor Network (WSN) is mostly used in real time applications, like as automobile traffic control, military purpose, medical area and environment examination. ZigBee is a Wireless Personal Area Network (WPAN) based on IEEE 802.15.4 wireless protocol. ZigBee gives short distance communication with low data rate, low power consumption and low complexity. In this paper the performance of hybrid topology is analyzed with OPNET Modeler 14.5 under the mobility of ZigBee End Devices (ZEDs) and ZigBee Coordinators (ZCs) using different trajectories in terms of load, traffic received and data drop. The Results show that when ZEDs and ZCs are move, the Mesh-Tree hybrid topology and Star-Tree hybrid topology gives best performance respectively. We also found that the performance of wireless sensor network for inner square and circle trajectories is superior to outer square trajectory.
\end{abstract}

\section{Keywords}

WSN, ZigBee, OPNET Modeler, Mobility, Trajectory.

\section{INTRODUCTION}

ZigBee was created by IEEE 802.15.4 task group and ZigBee alliance. To introduce wireless communication ZigBee cooperation makes some standards. This standard was produced to meet the accompanying important need of minimal cost, ultra low power utilization, utilization of unlicensed radio bands, simple implementation, adaptable and extendable systems [1, 3, and 7]. A WSN consists of many inexpensive wireless sensors, which are communicating with neighbouring nodes, processing environmental information, capable of collecting and storing. In the past, sensors are connected by wire lines. With the development of ad hoc networking technologies, tiny sensors can communicate through wireless links in a more convenient manner [2]. ZigBee works in the industrial, scientific and medicinal (ISM) radio band, particularly at $2.4 \mathrm{GHz}$ globally and $868 \mathrm{MHz}$ or $915 \mathrm{MHz}$ in particular parts of the world. IEEE 802.15.4/ZigBee architecture ZigBee characterizes two layers of the OSI (Open Systems Interconnection) demonstrate: the Application layer and the Network layer. Every layer performs a particular arrangement of administrations for the layer above. The diverse layers communicate through Service Access Points (SAP's) [2, 5, and 9]. These SAPs encase two sorts of substances: (1) an information substance (NLDESAP) to give information transmission administration and (2) a management element (NLME-SAP) giving all the management services between layers. The application layer (APL) provide interface between the ZigBee systems to its end users and also defines the device functionality. The basic function of this layer is to convert the input into digital data, and/or converts digital data into output. The ZigBee Network layer (NWK) is responsible for routing, network structure and security such as authentication, key management and encryption $[8,9]$. It additionally encases the neighbor tables and the Stockpiling of related data. The NWK layer gives one arrangement of interfaces, the Network Layer Data Entity Service Access Point (NLDE-SAP) used to exchange information with the APS $[10,11]$.

\subsection{ZigBee Device Object}

As to device part in the network, ZigBee characterizes three sorts of devices: ZigBee Coordinator (ZC), ZigBee Router (ZR) and ZigBee End Device (ZED) [12, 14].

\subsubsection{ZigBee Coordinator (ZC)}

ZigBee coordinator is responsible for setting up all the network parameters such as topology, packet size etc. It is a node with superior computing capabilities as compared to routers and end-devices. It is a gateway for the outside world to interact with the network. This role is generally assigned to the sink node $[11,15]$.

Coordinator Router End device

Fig 1: ZigBee Devices

\subsubsection{ZigBee Router (ZR)}

ZigBee router is the intermediary device in a network which transmits data from the source to the destination as well as senses the data from their adjoining environment $[3,9]$.

\subsubsection{ZigBee End Device (ZED)}

ZigBee end-device is capable of sensing data and completely depends on their parents (routers/coordinators) for routing their packets with least computing capabilities [6, 8].

\subsection{ZigBee Topologies}

The ZigBee standard permits the arrangement of three sorts of network topology: Star, Tree, and Mesh [12, 13].

\subsubsection{Star topology}

It is the least difficult of the three topologies, comprising of just a solitary coordinator with various end-devices as its children [11, 14]. 


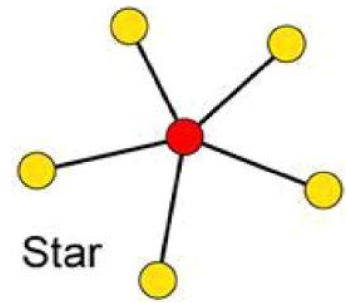

ZigBee Coordinator

ZigBee Devices

Fig 2: Star Topology

\subsubsection{Tree topology}

In this the devices compose themselves into a tree-like structure with the coordinator representing to the foundation of the tree, routers represent to the bases of sub-trees, and end-devices representing to leaves [7,9].

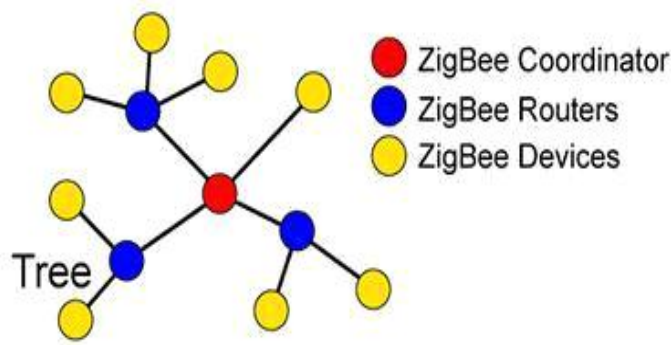

Fig 3: Tree Topology

\subsubsection{Mesh Topology}

In mesh topology routers and coordinators shape various connections among one another while having end-devices as their children's. Albeit more unpredictable in its development and operation, network topology is described by link/path excess which is known not in enhanced system power and system steering capacity $[12,15]$.

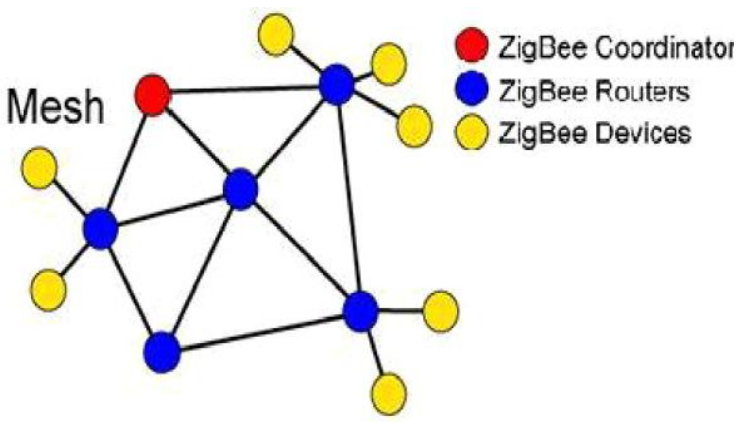

Fig 4: Mesh Topology

\subsubsection{ZigBee Hybrid Topologies}

ZigBee hybrid topologies are the combination of two or more different topologies. There are many different combinations of topologies but we have used Star-Tree (ST), Star-Mesh (SM) and Tree-Mesh (TM) as ZigBee hybrid topologies.

\subsection{ZigBee Coordinator and End Device Trajectories}

During the entire simulation duration the sink keeps on moving on the same path and trajectory of sink movement is along fixed path.

\subsubsection{Inner Square}

In this trajectory, the coordinator and end device move inside the network field at some distance from the boundary generally half the distance from the centre forming a square.

\subsubsection{Outer Square}

In this trajectory, the coordinator and end device move outside the network field on the boundary forming a square.

\subsubsection{Circle}

In this trajectory, the coordinator and end device move on almost a circular path [16].

\section{EXPERIMENTAL SETUP}

In this paper the effect of mobility of ZigBee End Devices and ZigBee Coordinators on hybrid topologies (Star-Mesh, StarTree and Mesh-Tree) is analyzed by using different trajectories (Inner Square, Outer Square and Circle). To make hybrid topology two PAN coordinators are used with different trajectories. The various scenarios are made by using simulation parameters given in table 1 . In this experiment firstly ZEDs are moving using different trajectories in each scenario under hybrid topologies and then ZCs are moving by using different trajectories in different scenarios under hybrid topologies. In each scenario there are 50 nodes which are placed randomly over an area of $100 * 100$ which are moving with the speed of $12 \mathrm{~m} / \mathrm{s}$ by using random way point model. In each scenario four ZRs are placed in each direction as shown in figure 5 which are fixed and two PAN coordinators is used. To get the performance of hybrid topology OPNET Modeler is used because OPNET Modeler provides a comprehensive development environment supporting the modeling of communication network and provides better environment for simulation, data collection and data analysis.

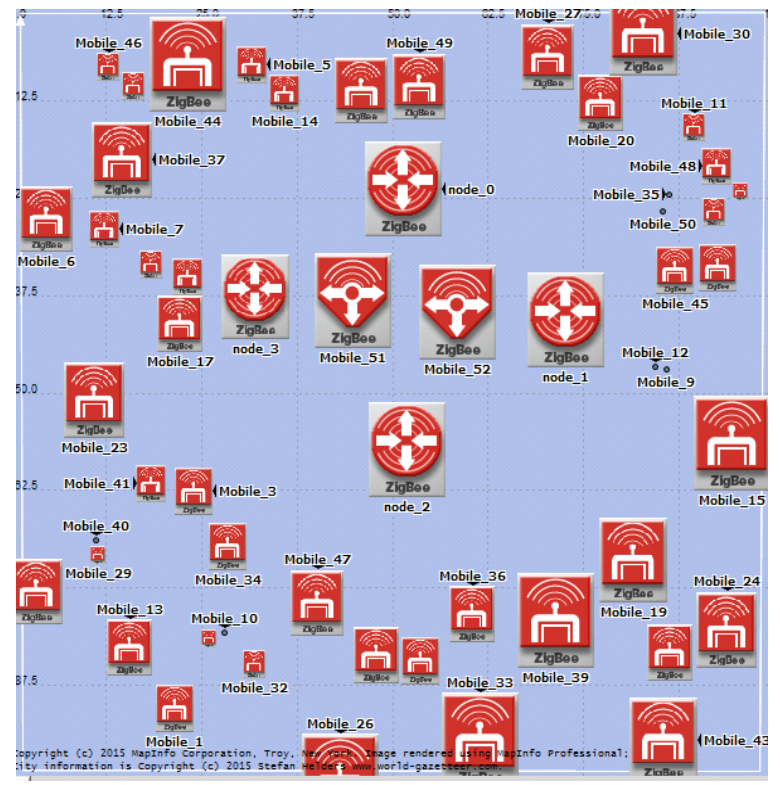

Fig 5: Scenario for Hybrid Topology 
Table 1: Simulation Parameters

\begin{tabular}{|c|c|}
\hline Parameters & Value \\
\hline Network Scale & $100 \mathrm{~m} * 100 \mathrm{~m}$ \\
\hline Number Of Nodes & 50 \\
\hline Network Type & Hybrid \\
\hline Mobility Model & Random Waypoint \\
\hline Speed of Mobile Nodes & $10 \mathrm{~m} / \mathrm{s}$ \\
\hline Pause Time & $100 \mathrm{~s}$ \\
\hline Trajectories & $\begin{array}{c}\text { Inner square, Outer } \\
\text { Square and Circle }\end{array}$ \\
\hline
\end{tabular}

\section{RESULTS AND DISCUSSIONS}

Simulation is conducted to evaluate the performance of hybrid topology under ZEDs and ZCs mobility by using different trajectories in terms of load, traffic received and packet dropped.

\subsection{Load}

Load represents the total load (in bits/sec) submitted to ZigBee 802.15.4 MAC by all higher layers in all wireless Personal Area Network (WPAN) nodes of the network. Figure 6, 7 and 8 shows the load variations of different hybrid topologies when ZigBee Coordinators and ZigBee End Devices are moving in circle, inner square and outer square trajectories. The graphs and Table 2, 3 shows that the load in ZigBee based wireless sensor network for MT hybrid topology is maximum under mobility of ZEDs in outer square trajectory and minimum under mobility of ZCs in inner square trajectory.

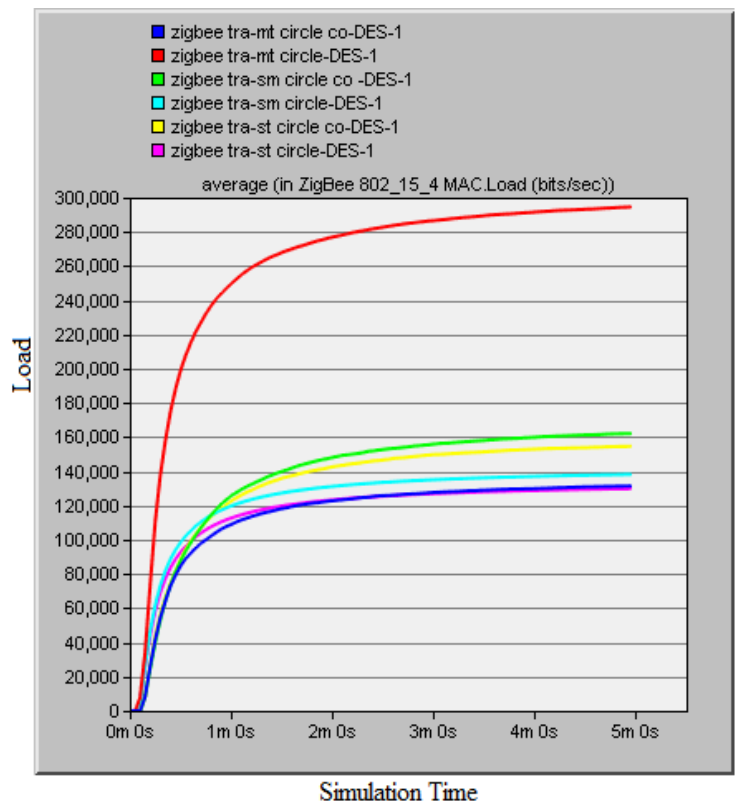

Fig 6: Load for Circle Trajectory

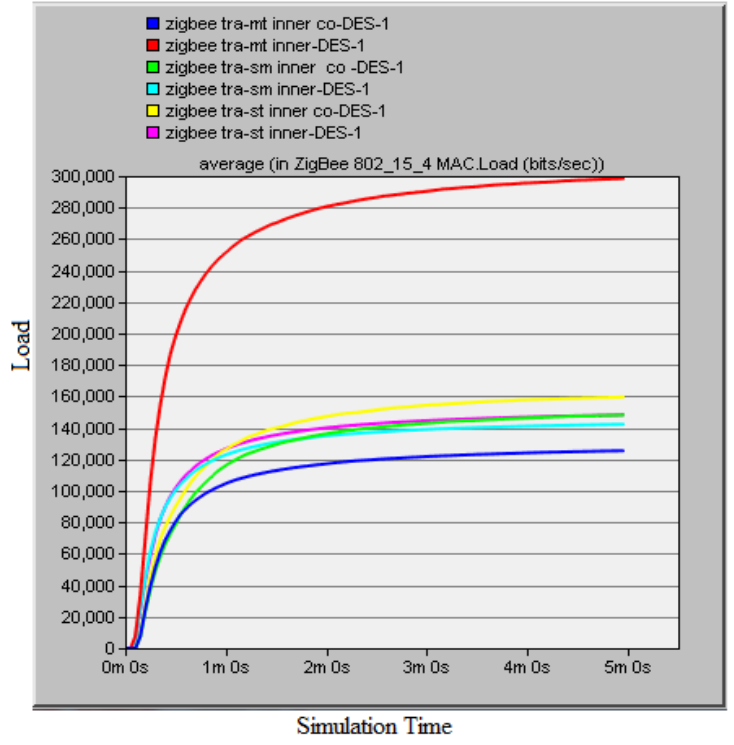

Fig 7: Load for Inner Square Trajectory

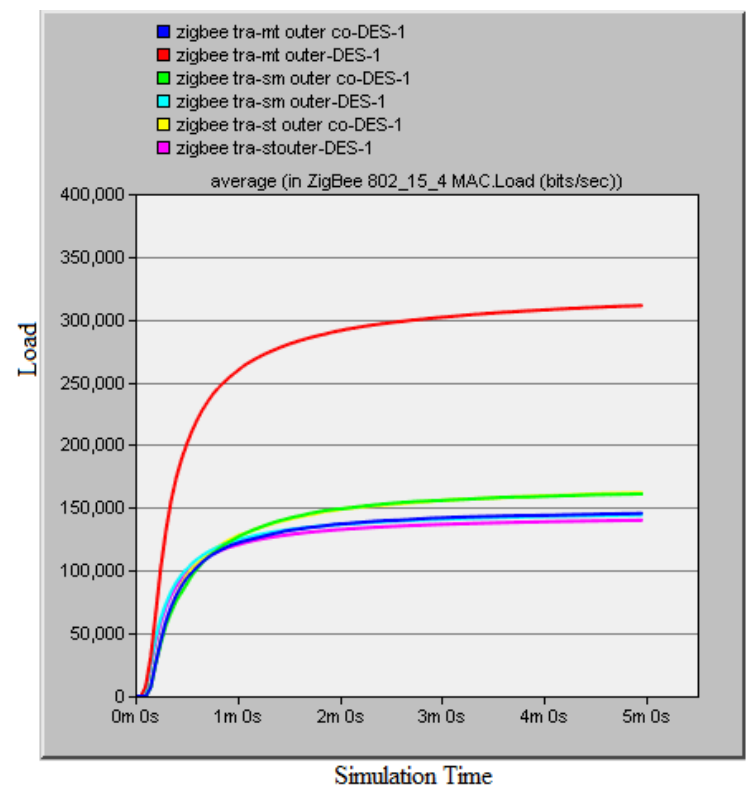

Fig 8: Load for Outer Square Trajectory

\subsection{Traffic Received}

Traffic received represents the total traffic successfully received by the MAC from physical layer in bits/sec. When these statistics are reported in units of bits/second, the physical and the MAC header sizes are included in the computation of the total amount of traffic received. These statistics record all the data received on the network interface regardless of the destination address. Graphs (Figure 9, 10 and 11) and Table 2, 3 describes the changes of traffic received with different hybrid topologies when ZigBee End Devices and ZigBee Coordinators are moving in circle, Inner Square and outer square trajectories. Traffic received in ZigBee based wireless sensor network for MT hybrid topology is maximum when ZEDs move in circle and inner square trajectories. When ST topology is used in the same network then minimum traffic is received under mobility of ZCs in circle trajectory. 


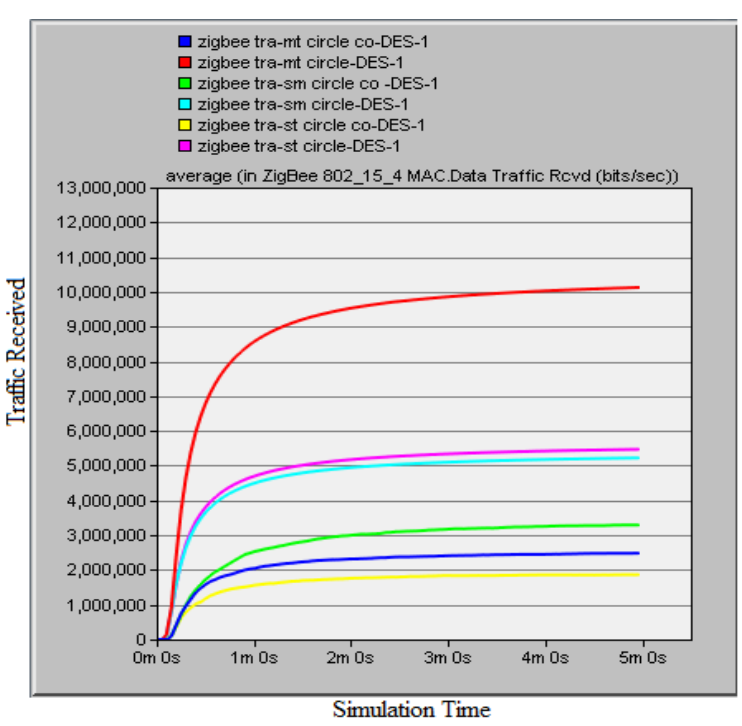

Fig 9: Traffic Received for Circle Trajectory

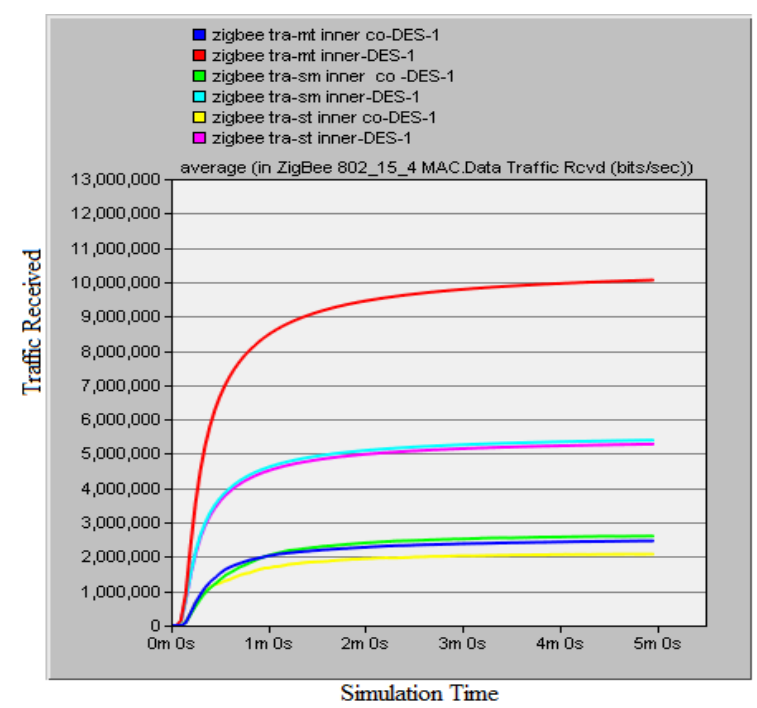

Fig 10: Traffic Received for Inner Square Trajectory

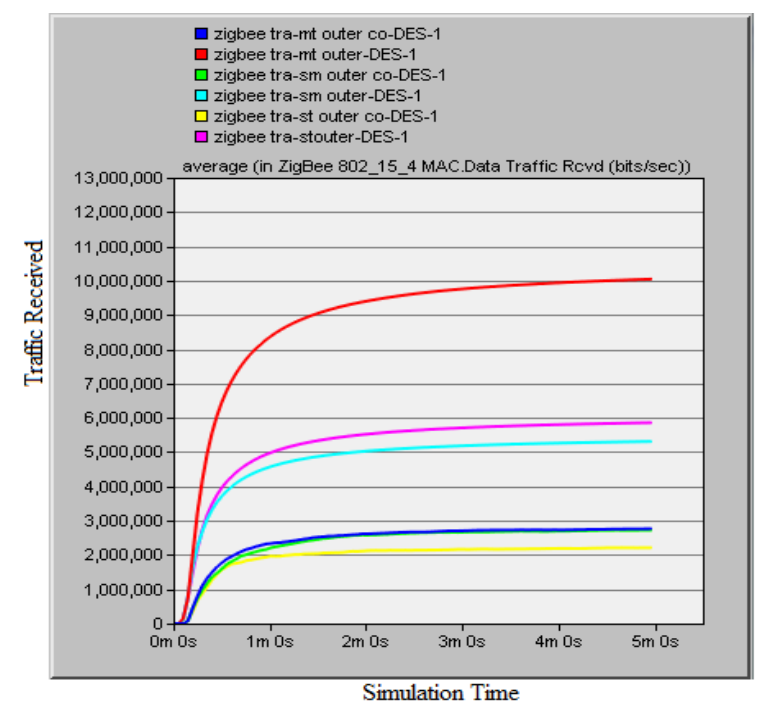

Fig 11: Traffic Received for Outer Square Trajectory

\subsection{Packet Drop}

Packet drop represents the drop by the layer due to not being joined to a network. This statistic records the total amount of data that was received from the upper layer and then dropped by all nodes in the network due to repeatedly failed retransmissions (i.e., exceeded the corresponding short retry or long retry threshold value). The packet drop rate of different hybrid topologies when ZigBee Coordinators and ZigBee End Devices are moving in outer square, circle and inner square trajectories are shown in figure 12, 13 and 14. It is found that minimum packets are dropped for ST topology when ZCs move in circle and inner square trajectories and for MT topology maximum packets are dropped under mobility of ZEDs in circle trajectory. The value of packet drop rate for hybrid topologies using different trajectories are mentioned in Table 2 and 3.

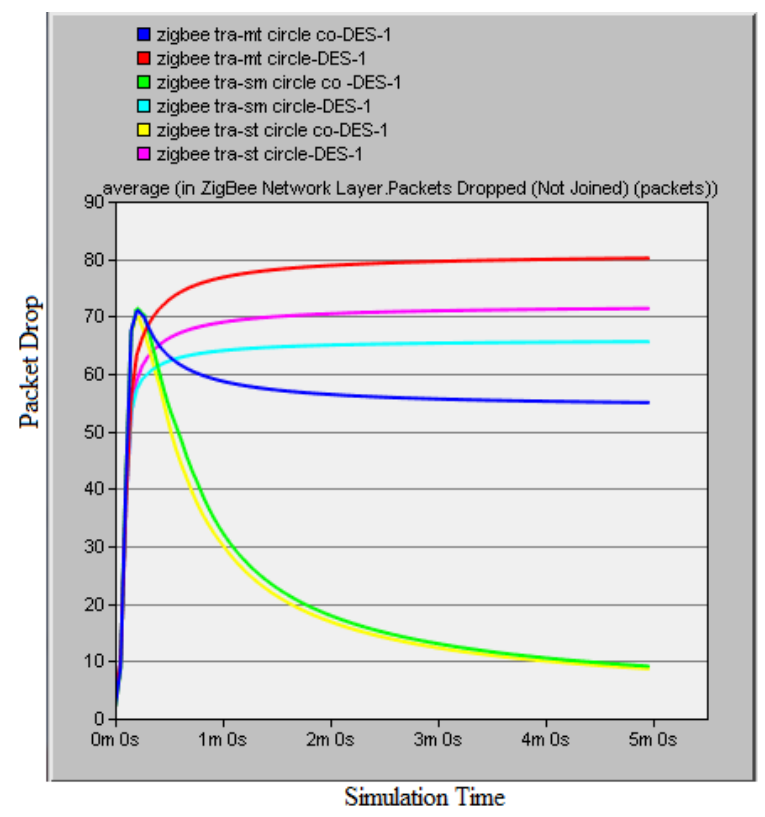

Fig 12: Packet Drop for Circle Trajectory

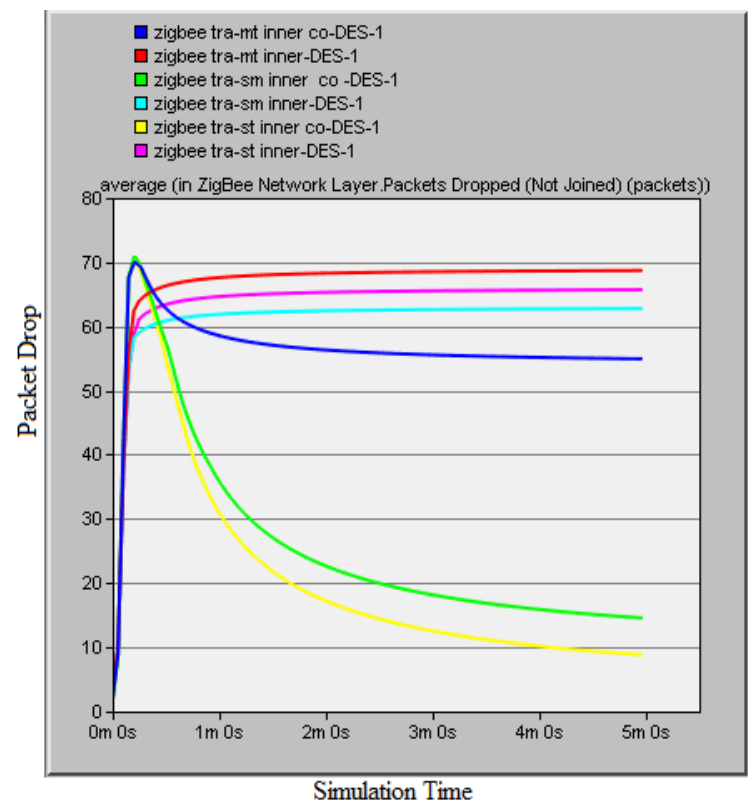

Fig 13: Packet Drop for Inner Square Trajectory 


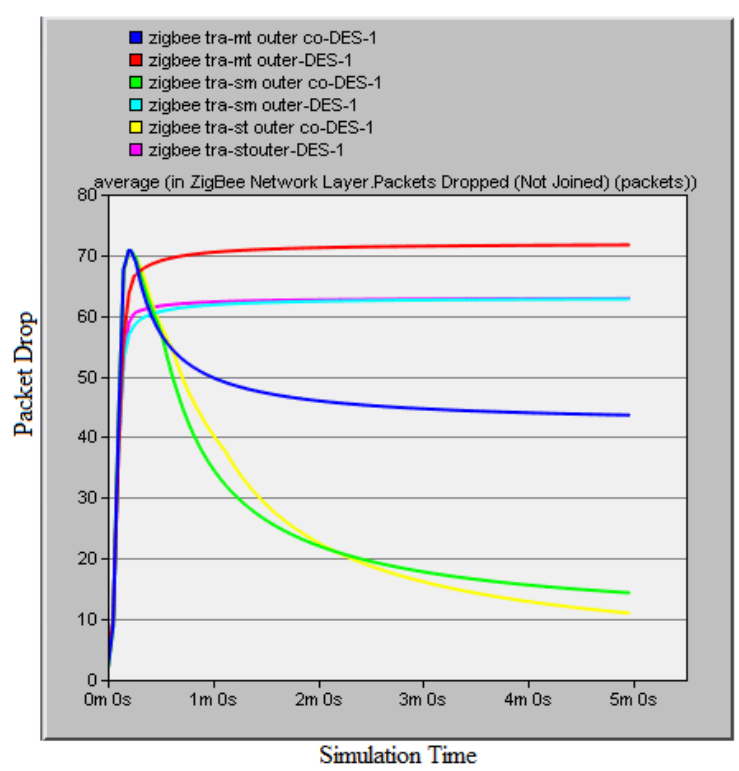

Fig 14: Packet Drop for Outer Square Trajectory

Table 2: Values of Different Parameters when ZEDs Move

\begin{tabular}{|c|c|c|c|c|}
\hline Trajectories & $\begin{array}{c}\text { Hybrid } \\
\text { Topologies }\end{array}$ & $\begin{array}{c}\text { Load } \\
\text { (bits/sec) }\end{array}$ & $\begin{array}{c}\text { Traffic } \\
\text { Received } \\
\text { (bits/sec) }\end{array}$ & $\begin{array}{c}\text { No. of } \\
\text { Packet } \\
\text { Drop }\end{array}$ \\
\hline \multirow{2}{*}{ Circle } & ST & 130000 & 5400000 & 72 \\
\cline { 2 - 5 } & MT & 298000 & 10100000 & 81 \\
\cline { 2 - 5 } & SM & 139000 & 5100000 & 65 \\
\hline \multirow{2}{*}{$\begin{array}{c}\text { Inner } \\
\text { square }\end{array}$} & ST & 148000 & 5200000 & 66 \\
\cline { 2 - 5 } & MT & 300000 & 10100000 & 69 \\
\cline { 2 - 5 } & SM & 141000 & 5300000 & 63 \\
\hline \multirow{2}{*}{$\begin{array}{c}\text { Outer } \\
\text { square }\end{array}$} & ST & 145000 & 5995000 & 62 \\
\cline { 2 - 5 } & MT & 310000 & 10050000 & 72 \\
\cline { 2 - 5 } & SM & 148000 & 5200000 & 62 \\
\hline
\end{tabular}

Table 3: Values of Different Parameters when ZCs Move

\begin{tabular}{|c|c|c|c|c|}
\hline Trajectories & $\begin{array}{c}\text { Hybrid } \\
\text { Topologies }\end{array}$ & $\begin{array}{c}\text { Load } \\
\text { (bits/sec) }\end{array}$ & $\begin{array}{c}\text { Traffic } \\
\text { Received } \\
\text { (bits/sec) }\end{array}$ & $\begin{array}{c}\text { No. of } \\
\text { Packet } \\
\text { Drop }\end{array}$ \\
\hline \multirow{2}{*}{ Circle } & ST & 158000 & 1800000 & 09 \\
\cline { 2 - 5 } & MT & 130000 & 2500000 & 55 \\
\cline { 2 - 5 } & SM & 161000 & 3300000 & 09 \\
\hline \multirow{2}{*}{$\begin{array}{c}\text { Inner } \\
\text { square }\end{array}$} & ST & 160000 & 2100000 & 09 \\
\cline { 2 - 5 } & MT & 122000 & 2190000 & 55 \\
\cline { 2 - 5 } & SM & 143000 & 2600000 & 15 \\
\hline \multirow{2}{*}{$\begin{array}{c}\text { Outer } \\
\text { square }\end{array}$} & ST & 162000 & 2210000 & 11 \\
\cline { 2 - 5 } & MT & 144000 & 2790000 & 43 \\
\cline { 2 - 5 } & SM & 162500 & 2730000 & 14 \\
\hline
\end{tabular}

\section{CONCLUSION}

In this paper the effect of mobility of ZEDs and ZCs with hybrid topologies under different trajectories are concluded. To analyse the effect, 50 nodes are placed randomly and each node moves at a speed of $12 \mathrm{~m} / \mathrm{s}$ by using random way point model. The performance is compared on basis of load, traffic received and data drop. The results show that when ZigBee End Devices are moving then Mesh-Tree hybrid topology gives best performance for outer square trajectory in term of load and for circle and inner square trajectories in terms of traffic received. Further it is found that when ZigBee coordinators are moving then Star-Tree hybrid topology performs best for circle and inner square trajectories in terms of packet drop. Overall, it is found that circle and inner square trajectories based ZigBee wireless sensor network perform better in comparison to outer square trajectory. In future performance of ZigBee based wireless sensor network with hybrid topologies can be analysed by applying more complex trajectories.

\section{REFERENCES}

[1] Francesca Cuomo et al. "Performance analysis of IEEE 802.15.4 wireless sensor networks: An insight into the topology formation process", Computer Networks 53 (2009) 3057-3075

[2] Kumar, H., Sarma, D., Kar, A. and Mall, R. "Energy Efficient Communication Protocol for a Mobile Wireless Sensor Network System" in International Journal of Computer Science and Network Security, Vol.9, No.2.

[3] Yu-Kai Huang et al., "A Comprehensive Analysis of Low-Power Operation for Beacon-Enabled IEEE 802.15.4 Wireless Networks" IEEE Transaction on Wireless Communications, VOL. 8, NO. 11, Nov 2009.

[4] Jae Yeol Ha et al., EHRP: Enhanced Hierarchical Routing Protocol for ZigBee Mesh Networks", IEEE Communications Letters, Vol. 11, No. 12, Dec 2007

[5] Benoît Latre et al. "Throughput and Delay Analysis of Unslotted IEEE 802.15.4", Journal of networks, vol. 1, no. 1, May 2006.

[6] Mahlknecht, S. "WSSN (Wireless Self-sustaining Sensor Network) Project", 2005.

[7] Stefano Chessa "Crash faults identification in wireless sensor networks" Computer Communications, Volume 25, Issue 14, 1 September 2002, Pages 1273-1282.

[8] Akyildiz, I. F., Su, Sankara subramaniam, W. Y. and Cayirci, E. "Wireless sensor networks: A survey," Computer Networks (Elsevier), vol. 38, pp. 393-422, 2002 .

[9] I.F. Akyildiz et al., "Wireless sensor networks: a survey Computer Networks, Volume 38, Issue 4,15 March 2002, Pages 393-422.

[10] Pottie, G. J. and Kaiser, W. J. "Wireless Integrated Network Sensors," Communications of the ACM, vol. 43, no. 5, pp. 551-558, May 2000. 
[11] Michimune Kohno et al., "An adaptive sensor network system for complex environments", Robotics and Autonomous Systems, Volume 28, Issues 2-3,31 August 1999, Pages 115-125.

[12] Helena Fernández-Lopez, "Towards the design of efficient nonbeacon-enabled ZigBee networks" Computer Networks 56, (2012) 2714 - 2725.

[13] Yasvindar Rana et al. "IEEE 802.15.4 based Investigation and Simulation Evaluation of ZigBee Tree and Mesh Topology using Different QoS" in IJCSMC, Vol. 3, Issue. 6, June 2014, pg.922 - 932.
[14] ELKISSANI Kaoutar et al. "ZigBee Routing OPNET Simulation for a Wireless Sensors Network" (IJACSA) International Journal of Advanced Computer Science and Applications, Vol. 5, No. 12, 2014.

[15] Gilbert E. PEREZ et al. Comparing a Real-Life WSN Platform Small Network and its OPNET Modeler model using Hypothesis Testing", SYSTEMICS, CYBERNETICS AND INFORMATICS VOLUME 12 NUMBER 7 - YEAR 2014 ISSN: 1690-4524.

[16] Harsh Dhaka, Atishay Jain, Karun Verma, "Impact of Coordinator Mobility on the throughput in a ZigBee Mesh Networks", IEEE, 2010. 